\title{
Experimental Study on Parkinson Disease Model of Rats
}

\author{
Cao Fei ${ }^{1 *}$, Sun Sheng-Gang ${ }^{1}$, Cao Xue-Bing ${ }^{1}$, Wang Tao ${ }^{1}$, Cheng Ji-Xiang ${ }^{1}$, Mei Yuan-Wu ${ }^{1}$, Tong E-Tang ${ }^{1}$ and Luo Fang ${ }^{2}$ \\ ${ }^{1}$ Department of Neurology, Union Hospital, Tong-ji Medical College, Hua-zhong Scientific and Technologic University, Wuhan, PR China \\ ${ }^{2}$ Trade and Financial Hospital of Hubei Province, Wuhan, PR China
}

\begin{abstract}
Objective: To observe the characteristics of praxiology of Parkinson disease (PD) rats. Methods: At 1d, $7 d$, 14d after PD rats' model were successful; we observed the markers of rotational behavior of Parkinson disease (PD) rats. For example: start time, continuous time, maximum turning, etc.

Results: From 1d to 14d, PD rats, start time of the rotational behavior elongated gradually, continuous time shortened by degrees, maximum rotational speed and the number of the rotational behavior were invariable.

Conclusions: Improved method of PD is scientific, reliable, and simple. Substantial nigral pathology of PD rats makes the foundation of the rotational behavior. The injury of substantial nigral can be reflected by maximum rotational speed and the number of the rotational behavior of PD rats.
\end{abstract}

Keywords: Parkinson disease; Rotational behavior

\section{Introduction}

Parkinson's disease is a common neurological degenerative disease in the elderly. PD pathogenesis remains mysterious. We often utilize 6-OHDA for a PD rat model. But there are injection site, quantity, and many other controvers. And it is still lack of a more detailed description of behavior after the success of the model. This experiment, by observing the characteristics of model's behavior change after successful improved PD rat, verifies the improved model of PD is scientific and reliable, and conducts a more detailed observation of behavior study.

\section{Materials and Methods}

\section{Animals selection and subgroup}

A total of 30 adult male Wister rats (Tongji Medical University Laboratory Animal Center), weighting $200 \mathrm{~g}$, were used. All model rats received 6-OHDA injection (6-OHDA injection: 6-OHDA、 vitamin C powder (sigma company), $0.3 \%$ 6-OHDA injection according to the ratio of 2.5:1 with saline. The injection was used when preparation and stored sealed, low temperature, and dark) stereotactic brain injection. After 6-OHDA administration $1 \mathrm{~d} 、 7 \mathrm{~d}$ and $14 \mathrm{~d}, 10$ rats per group were observed every time.

\section{Rat model of Parkinson's disease}

According to improved Thomas method to produce rat model of PD: after anesthetized with $7 \%$ chloral hydrate $(0.6 \mathrm{ml} / 100$ g) by intraperitoneal injection, rats were fixed in a brain threedimensional positioning instrument (manufactured by northwest optical instrument factory) horizontally. Maxillary incisor teeth plane was $2.4 \mathrm{~cm}$ lower than biauricular line plane (id est. the nose bar was $2.4 \mathrm{~cm}$ lower than biauricular line). Assurance anterior and posterior fontanel was at the same horizontal plane. Gently shaking in all directions proved head fixation reliable and then into the next step. The rats' hair on the top of the head was usually shaved. The operating field was disinfected by using iodine tincture or alcohol. Cutting the middle cranial scalp and fascia in length for $1.5 \mathrm{~cm}$, stripping the periosteum and exposuring the anterior fontanel. Refering to stereotaxic atlas of rats (Bao xinming etc.) determined the right substantia nigra, mesencephal gaster-tegmental's $3 \mathrm{D}$ coordinate location. SNc location: $4.8 \mathrm{~mm}$ after anterior fontanel, $2.0 \mathrm{~mm}$ at the right of the mean line, $7.5 \mathrm{~mm}$ under the dura mater; VTA location: $4.4 \mathrm{~mm}$ after anterior fontanel, $1.2 \mathrm{~mm}$ at the right of the mean line, $7.5 \mathrm{~mm}$ under the dura mater. Drilling two skull holes by electric cranial drill, drawing-off 0.36 -OHDA by a $10 \mu \mathrm{l}$ microinjector, every point injected $3.3 \mu$ l. Needling slowly when injection. Attention to the needle tip bevels for caudal, and the injection speed controls 0.5 $\mu \mathrm{l} / \mathrm{min}$. Stop for $10 \mathrm{~min}$ when injecting over and then withdrawal of needle slowly following $1.0 \mathrm{~mm} / \mathrm{min}$. Gelatin sponge filled skull hole, then sutured fascia and skin and painted the suture apartment with Ma Yinglong ointment. Rats should keep warm post operation, and put in cage to feeding after awake.

\section{Observational study of behavior}

At the next day's 8:00 of the scheduled time rats were tested for rotation in a diameter of $40 \mathrm{~cm}$ stainless steel basin. They were introduced $0.5 \mathrm{mg} / \mathrm{kg}$ APO of intraperitoneal injection. Observing time was $2 \mathrm{~h}$. The rotation number was measuring with bell-type measurement.

\section{Results}

\section{Behavioral analysis}

The average number of PD's rats rotating circle was no significant difference from $1 \mathrm{~d}$ to $14 \mathrm{~d}$. Typical rotation: beyond the control, clockwise direction to the contra lateral rotation, head and tail connected, equal rotation to the left hind limb as the fulcrum, many accompanied with hair erection simultaneously, rpm more than 7 times, the maximum speed of $61 \mathrm{rpm}$, the maximum speed no significant change, the start-up time slowed down gradually, the

*Corresponding author: Cao Fei, Department of Neurology, Union Hospital, Tongji Medical College, Huazhong University of Science and Technology, Wuhan, PR China, E-mail: caofyx@163.com

Received April 11, 2013; Accepted July 28, 2013; Published July 31, 2013

Citation: Fei C, Sheng-Gang S, Xue-Bing C, Tao W, Ji-Xiang C, et al. (2013) Experimental Study on Parkinson Disease Model of Rats. J Neurol Disord 2: 127 doi:10.4172/2329-6895.1000127

Copyright: (C) 2013 Fei C, et al. This is an open-access article distributed unde the terms of the Creative Commons Attribution License, which permits unrestricted use, distribution, and reproduction in any medium, provided the original author and source are credited. 
duration shorten gradually. The experimental result manifested that, for each group of Parkinsonian rat models, along with time increasing, the start-up time was slowed down gradually and the duration was shorten gradually, but the rotation speed and the maximum speed were not changed.

\section{Discussion}

\section{Preparation of rat model of Parkinson's disease}

Rat model of PD is a long cycle and the many influencing factors animal model. For a high degree of simulated and strong comparison model, we improve Thomas method [1] as follows: (1) 6-OHDA injection production: 6-OHDA、vitamin C powder (sigma company), $0.3 \%$ 6-OHDA injection according to the ratio of 2.5 : 1 with saline. The injection is used when preparation and stored sealed, low temperature, and dark. (2) Rats selection: select the same sex rats, weighing 180 200 g, and fix their heads by stereotaxis instruments. These rats's tolerance is stronger than that of normal ones. More importantly, after the success of the model, drug use and effect observation will not be affected because of the overweight and tolerance decrease of the rats. (3) Consider the synoptic influence before experiment. Create conditions as far as possible to keep room at the optimum temperature and humidity for the rats. (4) Identify no abnormal rotational behavior in rats before experiment. (5) There are four fixed-point injection nigrostriatum ways: one-point, two-point, three-point and four-point. Two-point injection is ideal method. It has good performance in aspects of ease of operation, degree of injury, survival rate, and rotation behavior and so on. This view is the same as Perry et al. [2]. (6) Stop bleeding by gelatin sponge after fixedpoint operation. Disinfect the suture by idodine tincture. Attention to keep warm. Record daily water intake, feed volume and weight of rats strictly when feed the model of rats. Keep forage clean, fresh and dry. (7) Certifying a successful model should combine behavior study with the substantia nigra pathology. (8) Surgical trauma should be as small as possible. It should be aseptic as far as possible during intraoperation. Paying attention to keep warm after operation could improve survival rates significantly. Neurotoxin MPTP (1-methyl-4phenyl-1, 2, 3, 6-tetrahydropyridune) was also common agentia used for rats of PD model [3]. It was reported that MPTP only suited for the production of dog, mouse model. Due to rats MPTP-resistant, it couldn't breakdown substantia nigra dopaminergic neurons [4]. More importantly early striatal DA neurons decreased over 70\% in mouse model of PD producing by MPTP. However, after 8-9 months, their striatal DA content could gradually restore. 6-OHDA had strong toxic effects on nerve endings. Compared with other rat model producing methods of PD, model by 6-OHDA had good degree of confidence, stable and irreversible performance in nigrostriatum morphology and rat ethology. (9). Experiment showed improved rat model of PD was reliable positioning, strong clinical mimesis and practicability, easy to operate features, etc.

\section{Ethologic character of PD rat model}

From $1 \mathrm{~d}$ to $14 \mathrm{~d}$, the average number of PD's rats rotating circle have no significant difference. Previous literatures on ethologic description

\begin{tabular}{|l|l|l|l|l|l|}
\hline Rats & Quantity & $\begin{array}{l}\text { Start-up } \\
\text { time }(\text { min) }\end{array}$ & $\begin{array}{l}\text { Max-circling } \\
\text { (rpm) }\end{array}$ & $\begin{array}{l}\text { Duration } \\
\text { (min) }\end{array}$ & $\begin{array}{l}\text { Circle number } \\
\text { (rev/30 min) }\end{array}$ \\
\cline { 3 - 6 } & & PD group & PD group & PD group & PD group \\
\hline 1d & 10 & $3 \pm 0.6$ & $56 \pm 2$ & $180 \pm 14$ & $237 \pm 20$ \\
\hline 5d & 10 & $10 \pm 2.2$ & $58 \pm 3$ & $137 \pm 13$ & $240 \pm 20$ \\
\hline 10d & 10 & $15 \pm 2.0$ & $59 \pm 2$ & $80 \pm 18$ & $239 \pm 19$ \\
\hline
\end{tabular}

Table 1: Rotational behavior observation data sheet of PD rats. of PD rats are mostly very simple. For a scientific, comparable rat model of PD, we observe rotational behavior of PD rats carefully. Their typical performance is: beyond the control, clockwise direction rotation to the uninjured side, head and tail connected, equal rotation to the left hindlimb as the fulcrum, many accompanied with hair erection simultaneously, rpm more than 7 times, the maximum speed of $61 \mathrm{rpm}$. This test is also observed that as time increases, more and more difficult to start-up and the duration rotation shorten gradually. But the maximum speed has no significant change in each successful PD model group. Now in this aspect it is lack of detailed reports. We believe that the duration of rotation and the maximum rotation speed can be used as the test index of PD rat's striatum nigrostriatia injury because of their good constant reliability [5]. However the start time, duration of rotation do not have good constancy, we suggest do not adopt them.

When DA deceases over $80 \%$ in nigrostriatia system, DA receptor will be hypersensitivity. After rats ingest L-dopa and APO, they perform for the rotational behavior to the contralateral. Because the rotating circle number of PD rats is positively correlated with the injury degree of DA neurons in substantia nigra, the number of rotation behavior usually reflect the degree of DA neurons of substantia nigra [6]. There is no significant difference of PD rat's rotation behavior between the groups tested from $1 \mathrm{~d} \sim 14 \mathrm{~d}$. The rotation behavior don't aggravate along with decrease of nigral apoptotic cells and Nissl cells. We consider that is because the DA receptors are reduced or arising tolerance. This point is similar to Trugman's [7]. We can surmise that the degree of injury in the substantia nigra of PD rats is inverse proportion with start-up time, but direct ratio with duration of rotation. That is, the duration of rotation is probably reflected in injury of PD rat substantia nigra cell.

\section{References}

1. Thomas J, Wang J, Takubo H, Sheng J, de Jesus S, et al. (1994) A 6-hydroxydopamine-induced selective parkinsonian rat model: further biochemical and behavioral characterization. Exp Neurol 126: 159-167.

2. Perry TL, Yong VW, Ito M, Foulks JG, Wall RA, et al. (1984) Nigrostriatal dopaminergic neurons remain undamaged in rats given high doses of L-DOPA and carbidopa chronically. J Neurochem 43: 990-993.

3. Chiueh CC, Markey SP, Burns RS, Johannessen JN, Pert A, et al (1984) Neurochemical and behavioral effects of systemic and intranigral administration of $\mathrm{N}$-methyl-4-phenyl-1,2,3,6-tetrahydropyridine in the rat. Eur J Pharmacol 100: 189-194.

4. Chiueh CC, Johannessen JN, Sun JL (1986) Reversibale neurotoxicity of MPTP in the nigrosreiatal dopaminergic system of mice. MPTP : A neurotoxin producing a Parkinsonian syndrome. Neuroscience 102: 473.

5. Tian X, Guan X, Liu B (1997) Effects of ganglioside on apomorphine-induced rotational behavior?dopamine content of striatum and pathological changes of substantia nigra in the rat models of Parkinson diease. Chin J Neurol 30: 208.

6. Henry B, Crossman AR, Brotchie JM (1998) Characterization of enhanced behavioral responses to L-DOPA following repeated administration in the 6-hydroxydopamine-lesioned rat model of Parkinson's disease. Exp Neurol 151: 334-342.

7. Trugman JM, Hubbard CA, Bennett JP Jr (1996) Dose-related effects of continuous levodopa infusion in rats with unilateral lesions of the substantia nigra. Brain Res 725: 177-183. 